\title{
TWENTY-FIFTH ANNUAL SASKATCHEWAN CHRISTMAS BIRD COUNT, 1966
}

compiled by Mary Houston, 863 University Drive, Saskatoon

Generally mild weather predominated during the entire count period and only two localities (Regina and Yorkton, the latter with a high of $-18^{\circ}$ ) encountered below zero temperatures on the day of their count.

Sixty species were recorded on count day (exceeded only in 1962-68 species, and $1960-61$ species) and eight additional species were seen during the count period.

The outstanding record this year was the Mountain Chickadee sighted by Steve Mann and party in the Piapot area. This was not only a new species for the Christmas count list, but a species never before sighted in Saskatchewan at any time of year and hence an addition to the provincial list. This bird appeared at the Mann feeding tray prior to December 11. The other new species for the Christmas count list was the Great Gray Owl seen near Cowan Lake, 23 miles north of Big River, and between Pas Trail and Forest Trail, between 30 and 12 miles northeast of Nipawin. These two species bring the all-time total for 25 Christmas counts to a remarkable 113 species.

We welcome participation by the new society at Swift Current and by the reactivated Yorkton society. Stan Riome helped organize no less than five counts at five localities in the Nipawin area and Steve Mann did the best job of organizing a rural area with 14 ranchers participating in his count south of Piapot. Manitoba counts were sent to the Manitoba Natural History Society Newsletter. Three Alberta and one N.W.T. count are appended to our list that follows. BATTLEFORD, Dec. 26; 30 miles by car and 3 hours on foot; temperature $25^{\circ}$; wind light, sky overcast; 12 inches of snow. 8 species, 128 individuals. Ruffed Grouse, 8; Rock Dove, 13; Hairy Woodpecker, 1; Black-billed Magpie, 3; Black-capped Chickadee, 4; Bohemian Waxwing, 85; House
Sparrow, 11; Pine Grosbeak, 3. (Add: Goshawk, 1, Dec. 28; Sharp-tailed Grouse, 4, Dec. 27; Gray Partridge, 9, Dec. 22; Great Horned Owl, 1, Dec. 28; Short-eared Owl, 1, Jan. 2; Common Redpoll, 6, Dec. 24.)-Harold Fisher, Spencer G. Sealy.

BROADVIEW, Dec. 26; 32 miles by car and $1 \frac{1}{2}$ miles on foot; temperature $0^{\circ}$; no wind; 7 species, 216 individuals. Ruffed Grouse, 2; Gray Partridge, 4; Black-billed Magpie, 17; Black-capped Chickadee, 10; Starling, 25; House Sparrow, 8; Snow Bunting, 150.Douglas G. Francis.

CARLEA, Dec. 27 ; temperature, $14^{\circ}$; wind S.E. $6 \mathrm{mph}$. 9 species, 70 individuals. Sharp-tailed Grouse, 4; Hairy Woodpecker, 2; Downy Woodpecker, 3; Gray Jay, 1; Blue Jay, 6; Blackcapped Chickadee, 9; House Sparrow, 14; Evening Grosbeak, 29; Slatecolored Junco, 2.-Mrs. R. C. Roney, Wesley Roney.

CODETTE, Dec. $27 ; 5$ miles in 6 hours on skis. 18 species, 135 individuals. Goshawk, 1; Ruffed Grouse, 3; Sharptailed Grouse, 6; Gray Partridge, 4; Rock Dove, 6; Great Horned Owl, 2; Snowy Owl, 2; Hairy Woodpecker, 1; Downy Woodpecker, 1; Black-billed Magpie, 5; Common Raven, 3 ; Blackcapped Chickadee, 4 ; Bohemian Waxwing, 11; Northern Shrike, 1; House Sparrow, 12; Evening Grosbeak, 16; Pine Grosbeak, 11; Snow Bunting, 46. (Add: Boreal Owl, Dec. 28; Common Redpoll, Dec. 25).-Wally Harstad.

CONNEL CREEK, Dec. 27; 7 hours, 10 miles on snowshoes in heavy black spruce stands; temperature $20^{\circ}$; wind N.W. $5 \mathrm{mph}$; partly cloudy with light intermittent snow. 10 species, 128 individuals. Ruffed Grouse, 1; Hairy Woodpecker, 2; Downy Woodpecker, 1; Black-billed Magpie, 2; Common Raven, 3; Black-capped Chickadee, 7; Red-breasted Nuthatch, 3; Starling, 1; Pine Grosbeak, 8; Snow Bunting, 100. -Paul Fowler. 
COWAN LAKE-COWAN RIVER, Dec. $31 ; 17$ miles by car and $1 / 2$ mile on foot in 2 hours; temperature $8^{\circ}$; overcast with light wind; 10 inches if snow. 8 species, 20 individuals. Great Gray Owl, 1; Hairy Woodhecker, 1; Black-billed Magpie, 1; Common Raven, 1; Black-capped Chickadee, 3; Boreal Chickadee, 1; Hoary Redpoll 1; Common Redpoll, 11. -Robert H. Buhr, C. Stuart Houston.

DILKE, Dec. 28; 52 miles by car and on foot in 3 hours; temperature $5^{\circ}$ o $13^{\circ}$; sunny; wind N.W. 5-10 mph; 12 inches of snow, drifted. 10 species, 131 individuals. Sharp-tailed Grouse, 10; Gray Partridge, 28; Rock Dove, Hairy Woodpecker, 1; Horned Lark, 3; Black-billed Magpie, 14; Bohemian Waxwing, 10 ; House Sparrow, 50; Rusty Blackbird, 2; Snow Bunting, 11. (Add: Golden Eagle, 1, Dec. 31; Snowy Owl, 1, Dec. 24; Sawwhet Owl, 1, Jan. 1; Slate-colored Junco 1, Dec. 21, 22, 23, 25, 26.)Boswell Belcher (compiler), Margaret Belcher, Mr. and Mrs. S. R. Belcher. ERINFERRY, Dec. 21; light breeze with light snow; at feeding station. 6 species, 26 individuals. Hairy Woodpecker, 2; Downy Woodpecker, 2; Gray Jay, 4; Blue Jay, 12; Blackhilled Magpie, 2; Black-capped Chickadee, 4. (Add: Sharp-tailed Grouse, 9, Dec. 22; Common Raven, 2, Dec. 22, 25; Evening Grosbeak, 28, Dec. 22; Snow Bunting, large flock, Dec. 22).Mrs. E. A. Dodd.

FORT QU'APPELLE, Dec. 31; temperature about $10^{\circ} ; 8$ inches of snow. 5 species, 66 individuals. Downy Woodpecker, 1; Black-billed Magpie, 11; Black-capped Chickadee, 3 ; Northern Shrike, 1; House Sparrow, 50. (Add: Sharp-tailed Grouse, 11, Jan. 2; Snowy Owl, 1, Dec. 29; Hairy Woodpecker, 2, Dec. 30 and Jan. 1; Blue Jay, 1, Dec. 22; Bohemian Waxwing, 16, Dec. 23, 40, Dec. 27, and 1, Dec. 28; Pine Grosbeak, 1, Dec. 30; Snow Bunting, 8, Dec. 25 and 10, Dec. 26).-E. Manley Callin (compiler), Mr. and Mrs. Errol Cochrane, Mr. and Mrs. Wm. Gray, Dale Nostbakken, Horace Reed.
HAWARDEN, Dec. 23; trip of 20 miles and around farm; 12 inches of snow. 4 species, 221 individuals. Gray Partridge, 15; Rock Dove, 3; Blackbilled Magpie, 3; House Sparrow, 200. (Add: Snowy Owl, 1, Dec. 31; Bohemian Waxwing, 2, Dec. 29).-Harold Kvinge.

INDIAN HEAD, Dec. 27; 4 miles by foot and 16 miles by car, also around farmyard, a total of 6 hours; temperature $15^{\circ}$; wind $\mathrm{S}$. at $10 \mathrm{mph}$.; overcast. 8 species, 283 individuals. Sharp-tailed Grouse, 4; Gray Partridge, 14; Great Horned Owl, 1; Downy Woodpecker, 1; Black-billed Magpie, 8; Black-capped Chickadee, 5 ; House Sparrow, 100; Snow Bunting, 150. (Add: Pigeon Hawk, 2, Dec. 23; Rock Dove, 3, Dec. 29; Hairy Woodpecker, 1, Dec. 29).- L Lorne Scott.

KELVINGTON - HIGH HILL, Jan. 2; 45 miles by car and about town in 3 hours; temperature $10^{\circ}$; clear. 8 species, 149 individuals. Downy Woodpecker, 1; Black-billed Magpie, 7; Common Raven, 1; Black-capped Chickadee, 2; Northern Shrike, 1; House Sparrow, 46; Evening Grosbeak, 35; Snow Bunting, 56. (Add: Pileated Woodpecker, 1, Dec. 28; Hairy Woodpecker, 1, Dec. 23; Bohemian Waxwing, 12, Dec. 21.)-Steve Naomi, Verna and Brian Waycheshen, Anton Waycheshen.

KENASTON, Dec. 28; temperature $15^{\circ}$; wind light; mostly clear; 12 inches of snow. 6 species, 28 individuals. Gray Partridge, 6; Great Horned Owl, 1; Black-billed Magpie, 11; House Sparrow, 5; Pine Grosbeak, 1; Snow Bunting, 4. (Add: Sharptailed Grouse, 16, Dec. 30 and 2, Dec. 27; Rock Dove, 26; Horned Lark, 3, Dec. 29; Common Redpoll, 4, Dec. 30.) -Lawrence Beckie.

LA LOCHE, Dec. $30 ; 8$ miles by dogteam and return in 5 hours and 18 miles by car in 3 hours; temperature $4^{\circ}$ to $16^{\circ}$; wind E. $10 \mathrm{mph}$.; hazy with intermittent sunshine; 14 inches of snow, unusually deep. 6 species, 30 individuals. Spruce Grouse, 1; Willow Ptarmigan, 6; Gray Jay, 2; Common 
Raven, 3; House Sparrow, 11; Common Redpoll, 7. (Add: Black-billed Magpie, 1, Dec. 29.) - C. Stuart Houston, Alfred Herman (Chipewyan guide).

MOOSE JAW, Dec. 26; 87 miles by car and 17 party miles on foot; temperature $10^{\circ}$ to $15^{\circ}$; wind light; overcast with light snow; 4 to 6 inches of snow on ground. 13 species, 2320 individuals. Sharp-tailed Grouse, 1; Ringnecked Pheasant, 30; Gray Partridge, 59; Rock Dove, 27; Great Horned Owl, 5; Snowy Owl, 5; Downy Woodpecker, 2; Black-billed Magpie, 95; Blackcapped Chickadee, 16; Bohemian Waxwing, 559; Northern Shrike, 1; Starling, 20; House Sparrow, 1,500. (Add: Hairy Woodpecker, 1, Dec. 27; Horned Lark, 50, Jan. 2; Rusty Blackbird, 2, Jan. 2; Pine Grosbeak, 9, Dec. 29; Slate-colored Junco, 12, Dec. 21; Snow Bunting, 125, Jan. 2.)-Mr. and Mrs. Peter Brodie, Dawn Joanne, Scott and Robin Brodie, Russell Dennison, Mr. and Mrs. Carl Ellis, John Horton, Mrs. Vesta Humphries, Miss Pat Kern, Mr. and Mrs. Cy Knight, Mrs. A. W. McAvoy, Mrs. Dorothy Rhodes, Michael Rhodes, Miss M. Ritchie, Mr. and Mrs. G. Silversides and Ruth, Mrs. F. B. Taylor. (Members of the Moose Jaw Natural History Society. Leith Knight, compiler.)

NIPAWIN, Dec. 27; 100 miles by car, 4 miles on snowshoes in $7 \frac{1}{2}$ hours; temperature $20^{\circ}$; calm to $5 \mathrm{mph}$. N. wind; overcast; 14 inches of snow. 17 species, 1020 individuals. Mallard, 2; American Goldeneye, 34; Hairy Woodpecker, 3; Downy Woodpecker, 3; Gray Jay, 3; Blue Jay, 2; Black-billed Magpie, 56; Common Raven, 49; Black-capped Chickadee, 8; Boreal Chickadee, 1; Starling, 32; House Sparrow, 200; Rusty Blackbird, 1; Evening Grosbeak, 28; Pine Grosbeak, 46; Common Redpoll, 2; Snow Bunting, 550. (Add: Ruffed Grouse, Jan. 2; Red Crossbill, 2, Dec. 28.)-David Riome. Stan Riome.

PAS TRAIL, Dec. 27; about yard in the a.m. and 43 miles by car in 3 hours in the p.m.; temperature $10^{\circ}$; wind calm; overcast with light snow; 14 species, 225 individuals. Goshawk,
2; Ruffed Grouse, 1; Sharp-tailed Grouse, 3; Great Gray Owl, 1; Hairy Woodpecker, 1; Downy Woodpecker, 1; Blue Jay, 6; Black-billed Magpie, 4; Common Raven, 9; Black-capped Chickadee, 4; Bohemian Waxwing, 25; Evening Grosbeak, 6; Pine Grosbeak, 22; Common Redpoll, 140.-Mrs. Anne Matthew, Mr. and Mrs. Walter Matthews, William Matthews.

PIAPOT, Dec. 28; 60 miles by car and 12 miles on foot in 26 party hours: temperature $24^{\circ}$; wind $12-15 \mathrm{mph}$; overcast; about $1 / 2$ inch of snow. 19 species, 736 individuals. Golden Eagle, 4; Sharp-tailed Grouse, 104; Ringnecked Pheasant, 3; Gray Partridge, 42; Great Horned Owl, 5; Snowy Owl, 2; Hairy Woodpecker, 2; Downy Woodpecker, 2; Horned Lark, 22; Black-billed Magpie, 79; Black-capped Chickadee, 51; Mountain Chickadee, 1; Bohemian Waxwing, 23; House Sparrow, 281; Pine Grosbeak, 26; Common Redpoll, 53; Tree Sparrow, 1; Song Sparrow, 1; Snow Bunting, 34. (Add: Brewer's Blackbird, 1, Dec. 23.)-Stanley Birchall, Henry and Edith Borman, Mrs. Mary Drever, Mrs. Phyllis Flagg, Leslie and Randy Flagg, Mr. and Mrs. Robert Mann and Patricia, Mr. and Mrs. Steve Mann (compiler), Peter Swain, Harry Williams.

RAYMORE, Dec. 28; 6 species, 86 individuals. Rock Dove, 11; Downy Woodpecker, 2; Black-billed Magpie, 4; Black-capped Chickadee, 6; House Sparrow, 49; Pine Grosbeak, 14. (Add: Ruffed Grouse, 2, Dec. 27; Sharptailed Grouse, 6, Dec. 30; Rosebreasted Grosbeak, 3, Dec. 25; Evening Grosbeak, 5, Jan. 1; Snow Bunting, 11. Dec. 29.) - Wayne Harris. REGINA, Dec. $26 ; 406$ miles by car in $27 \frac{1}{2}$ party hours and 23 miles on foot in $18 \frac{1}{2}$ party hours; temperature $-3^{\circ}$ to $11^{\circ}$; wind S.E. $12 \mathrm{mph}$.; overcast with light snow and mist over Waterfowl Park; 5 inches of crusted snow on open fields. 36 species, 4343 individuals. Mute Swan, 8; Whistling Swan, 5; Canada Goose, 350; Mallard, 450; Gadwall, 1; Pintail, 1; Bluewinged Teal, 1; Canvasback, 2; Lesser Scaup, 13; Common Goldeneye, 2; 
Ruddy Duck, 6; Ruffed Grouse, 1; Sharp-tailed Grouse, 10; Gray Partidge, 129; American Coot, 8; Rock Dove, 108; Great Horned Owl, 4; Snowy Owl, 9; Short-eared Owl, 1; Yellow-shafted Flicker, 3; Downy Woodpecker, 4 ; Horned Lark, 1 ; Blackbilled Magpie, 81; B la ck-capped Chickadee, 14; Boreal Chickadee, 2; Red-breasted Nuthatch, 1; Brown Creeper, 1; Robin, 3; Townsend's Soliaire, 1; Golden-crowned Kinglet, 12 ; Bohemian Waxwing, 1032; Northern Shrike, 3; Starling, 123; House Sparow, 1487; Pine Grosbeak, 3; Snow Bunting 463.-George Allen, Mrs. J. 8. Bailey, Margaret Belcher, Greg Bobbitt, Frank Brazier, Bill Brownlee, Eric Cooke, Mrs. Elizabeth Cruickhank, Doug Gilroy, Jim Jowsey, Dr. and Mrs. G. F. Ledingham, Sandra Ledingham, Christine MacDonald, Helen Morrison, Sally Moss, Mr. and Mrs. Charles Payne, Maureen Rever, Tom Riffel, Mr. and Mrs. Hugh Smith (compilers), Elisabeth Wagner, Dick Yeaton. (Regina Natural History Society.)

SASKATOON, Dec. $26 ; 226$ miles by car in $23 \frac{1}{2}$ party hours and 19 miles on foot in 12 party hours; temperacure $13^{\circ}$; wind S.E. 4 mph.; 24 species, 5929 individuals. Mallard, 33; Pintail, 1; Redhead, 1; Lesser Scaup, 4; Common Goldeneye, 10; Pigeon Hawk, 1; Ruffed Grouse, 1; Sharp-tailed Grouse, 74; Ring-necked Pheasant, 6; Gray Partridge, 100; Rock Dove, 261; Great Horned Owl, 6; Snowy Owl, 1; Hairy Woodpecker, 2; Downy Woodpecker, 5; Blue Jay, 15; Black-billed Magpie, 218; Black-capped Chickadee, 61; Bohemian Waxwing, 923; Northern Shrike, 2; Starling, 63; House Sparcow, 4027; Pine Grosbeak, 64; Common Redpoll, 50. (Add: Goshawk, 1, Jan. 1; Saw-whet Owl, 1, Dec. 29, 30, 31; Horned Lark, 2, Jan. 2; Cedar Waxwing, 1, Jan. 1; Slate-colored Junco, 1, Jan. 1; Snow Bunting, 27, Dec. 31.) -W. J. Bruchs, John Cartwright, Pern Cordery, R. V. Folker, Glen A. Fox, Dr. and Mrs. J. B. Gollop, Stuart Golly, Mr. and Mrs. J. Greaves, Mr. and Mrs. Vic Harper, Mr. and Mrs. Jim Hogg, Dr. and Mrs.
Stuart Houston, David Houston, Mr. and Mrs. Frank Lane, Elizabeth and Frances Lane, Mr. and Mrs. Murray Martin, Arnold Nijssen, W. S. Richards, Miss Elmire Sansregret, John and Stanley Shadick, Jim Slimmon, Keith Smilley, Allen Smith, Jim Wedgwood.

SPIRIT LAKE, Jan. 1; on foot for 6 hours; temperature $5^{\circ}$ to $30^{\circ}$; clear becoming overcast with strong wind; 15 inches of snow. 15 species, 145 individuals. Ruffed Grouse, 3; Sharptailed Grouse, 6; Hairy Woodpecker, 6; Downy Woodpecker, 9; Blackbacked Three-toed Woodpecker, 1; Blue Jay, 4; Black-billed Magpie, 3; Black-capped Chickadee, 55; Whitebreasted Nuthatch, 1; Bohemian Waxwing, 12; House Sparrow, 23; Evening Grosbeak, 3; Pine Grosbeak, 13; Common Redpoll, 4; Snow Bunting, 2. (Add: Great Horned Owl, 1; Dec. 25, 26, 27 and 30.)-Iris Anaka, Wm. Anaka, Joyce Gunn (compiler), Mrs. Gunn.

SWIFT CURRENT, Dec. 30; 10 miles by car and on foot in 2 hours; temperature $30^{\circ}$; wind light. 6 species, 67 individuals. Ring-necked Pheasant, 1; Gray Partridge, 17; Snowy Owl, 1; Black-billed Magpie, 4; Bohemian Waxwing, 30; Pine Grosbeak, 14 (Add: Rock Dove, 33, Dec. 27; Blackcapped Chickadee, 10, Jan. 1; Starling, 10, Dec. 27; House Sparrow, 300, Dec. 27.) - Marion Hare, Lillian McBean, Jean McDaid, Paul Nodge.

VAL MARIE, Dec. 26; 50 miles by car and 4 on foot; heavy overcast; cool following chinook conditions; 10 inches of snow. 15 species, 144 individuals. Rough-legged Hawk, 3; Prairie Falcon, 1; Ring-necked Pheasant, 3; Gray Partridge, 15; Great Horned Owl, 1; Snowy Owl, 1; Horned Lark, 50; Black-billed Magpie, 18; Bohemian Waxwing, 13; Starling, 6; House Sparrow, 1; Red-winged Blackbird, 4; Rusty Blackbird, 8; Tree Sparrow, 6; Snow Bunting, 5. (Add: Golden Eagle, 2, Dec. 27; Sharp-tailed Grouse, 1, Dec. 27; Common Crow, 1, Dec. 27; Black-capped Chickadee, 3, Dec. 27; Northern Shrike, 2, Dec. 27.) -J. 
David Chandler, Ruth Chandler.

WOODROW, Dec. 22; 44 miles in vicinity of Wood River and Pinto Creek; temperature $30^{\circ}$; calm; little snow. 10 species, 243 individuals. Golden Eagle, 1; Ring-necked Pheasant, 17; Gray Partridge, 59; Rock Dove, 5; Snowy Owl, 3; Short-eared Owl, 1; Horned Lark, 24; Black-billed Magpie, 3; House Sparrow, 30; Snow Bunting, 100.-C. H. Shulver.

YELLOW CREEK, Dec. 27; 4 miles on foot about yards, fields and wooded areas in 3 hours; temperature $6^{\circ}$ to $10^{\circ}$; calm; cloudy with light snow; 15 inches of snow. 9 species, 87 individuals. Gray Partridge, 4; Great Horned Owl, 1; Hairy Woodpecker, 1; Downy Woodpecker, 2; Black-billed Magpie, 3; Black-capped Chickadee, 9; House Sparrow, 52; Pine Grosbeak, 5; Common Redpoll, 10. (Add: Ruffed Grouse, 5, Dec. 23; Gray Jay, 1, Jan. 1; Bohemian Waxwing, 11, Jan. 1; Snow Bunting, 1, Dec. 23.)-Bohdan Pylypec.

YORKTON, Dec. $26 ; 30$ miles by car, 8 miles on foot; temperature $-18^{\circ}$; calm; heavy hoarfrost; $1 \frac{1}{2}$ feet of snow. 8 species, 423 individuals. Sharp-tailed Grouse, 16; Gray Partridge, 27; Rock Dove, 2; Hairy Woodpecker, 1; Black-billed Magpie, 8; Black-capped Chickadee, 9; House Sparrow, 273; Snow Bunting, 87.Michael Bantjes, Rod Bantjes, Dale Esopenko, Kent Hocevar, Glen Meekma, Larry Morgotch (compiler), Bob Pearce, Brian Procyshen, Jeff Smith, Frank Switzer, Gillean Switzer, Dr. Harold Wilkinson.

BLUFFTON, Alta., Jan. 2; 9 species, 225 individuals. Ruffed Grouse, 1; Gray Jay, 2; Blue Jay, 5; Black-billed Magpie, 1; Black-capped Chickadee, 4; House Sparrow, 2; Evening Grosbeak, 8; Pine Grosbeak, 2; Snow Bunting, 200. (Add: Hairy Woodpecker, 1, Dec. 27; Boreal Chickadee, 2, Dec. 25; 1 , Dec. 28; 2, Dec. 31.)-Fred Schutz.

STETTLER, Alta., Dec. 22; about barn and feed yards; temperature $20^{\circ}$; sunny; 5 inches of snow. 6 species, 96 individuals. Sharp-tailed Grouse, 1; Blue Jay, 1; Black-billed
Magpie, 5; Black-capped Chickadee, 5; Robin, 1; House Sparrow, 83. (Add: Ruffed Grouse, 4, Dec. 30; Gray Partridge, 8, Dec. 23; Great Horned Owl, 1, Dec. 31; Hairy Woodpecker, 1, Dec. 23; Downy Woodpecker, 1, Dec. 24; Bohemian Waxwing, 2, Jan. 2; Northern Shrike, 1, Jan. 1; Evening Grosbeak, 1, Dec. 29.)-Lloyd Lohr and Laverne Lohr.

WETASKIWIN, Alta., Jan. 2; all day about the farm; temperature, $20^{\circ}$; partly cloudy; 5 inches of snow. 5 species, 51 individuals. Hairy Woodpecker, 1; Blue Jay, 2; Black-billed Magpie, 3; Black-capped Chickadee, 5; House Sparrow, 40. (Add: Downy Woodpecker, 1, Dec. 26; Bohemian Waxwing, 7, Dec. 21; Evening Grosbeak, 1, Dec. 28; Pine Grosbeak, 1, Dec. 28.)—Fred H. Pegg.

FORT SMITH, N.W.T., Dec. 29; 40 miles by car in 4 hours; temperature $8^{\circ}$; wind light; overcast. 5 species, 94 individuals. Spruce Grouse, 1; Blackbacked Three-toed Woodpecker, 1; Gray Jay, 4; Common Raven, 83; Common Redpoll, 5. (Add: Starling, 2 -wintering here, for the first time this far north.) -Elsie and Ernie Kuyt, Hilah and Norman Simmons.

\section{ADDENDA TO PRAIRIE NEST RECORDS SCHEME SUMMARY}

Two species, for which nest records have been submitted, were omitted from the eight year summary of the P.N.R.S. which appeared in the December 1966 Blue Jay. In addition to those reported, the P.N.R.S. contains 54 cards on the Savannah Sparrow and three cards on the Baird's Sparrow. The addition of these two species to the summary brings the total number of species up to 238 and the total number of cards to 7,278 .

Three cards representing the nests of Yellow Rails in Manitoba were submitted in 1962 by Mr. J. Lane of Brandon. They were reponted in the 1962 report of the P.R.N.S. (Blue Jay, March 1963). However, the present whereabouts of these records is unknown. Can anyone help locate them? -Robert $R$. Taylor, University of Saskatchewan, Regina. 